Acta Univ. Sapientiae, Informatica, 5, 2 (2013) 271-287

DOI: 10.2478/ausi-2014-0014

\title{
Linear programming over exponent pairs
}

\author{
Andrew V. LELECHENKO \\ I. I. Mechnikov Odessa National University, Russia \\ email: $1 @ d x d y \cdot r u$
}

\begin{abstract}
We consider the problem of the computation of $\inf _{p} \theta p$ over the set of exponent pairs $\mathrm{P} \ni p$ under linear constrains for a certain class of objective functions $\theta$. An effective algorithm is presented. The output of the algorithm leads to the improvement and establishing new estimates in the various divisor problems in the analytical number theory.
\end{abstract}

\section{Introduction}

Exponent pairs are an extremely important concept in the analytical number theory. They are defined implicitly.

Definition 1 ([8, Ch. 2]) A pair $(\mathrm{k}, \mathrm{l})$ of real numbers is called an exponent pair if $0 \leqslant \mathrm{k} \leqslant 1 / 2 \leqslant \mathrm{l} \leqslant 1$, and if for each $\mathrm{s}>0$ there exist integer $\mathrm{r}>4$ and real $\mathrm{c} \in(0,1 / 2)$ depending only on $\mathrm{s}$ such that the inequality

$$
\sum_{a<n \leqslant b} e^{2 \pi i f(n)} \ll z^{k} a^{l}
$$

holds with respect to $\mathrm{s}$ and $\mathrm{u}$ when the following conditions are satisfied:

$$
u>0, \quad 1 \leqslant a<b<a u, \quad y>0, \quad z=y a^{-s}>1
$$

$f(t)$ being any real function with differential coefficients of the first $r$ orders in $[\mathrm{a}, \mathrm{b}]$ and

$$
\left|f^{(v+1)}(t)-y \frac{d^{v}}{d t^{v}} t^{-s}\right|<(-1)^{v} c y \frac{d^{v}}{d t^{v}} t^{-s}
$$

for $\mathrm{a} \leqslant \mathrm{t} \leqslant \mathrm{b}$ and $0 \leqslant v \leqslant \mathrm{r}-1$.

Computing Classification System 1998: G.1.6, F.2.1.

Mathematics Subject Classification 2010: 90C05, 11Y16, 11L07, 11N37

Key words and phrases: linear programming, exponent pairs, divisor problem 
But for the computational purposes more explicit construction is needed.

Proposition 2 The set of the exponent pairs includes a convex hull conv $\mathrm{P}$ of the set $\mathrm{P}$ such that

1. $\mathrm{P}$ includes a subset of initial elements $\mathrm{P}_{0}$, namely

(a) $(0,1)[8]$,

(b) $(2 / 13+\varepsilon, 35 / 52+\varepsilon),(13 / 80+\varepsilon, 1 / 2+13 / 80+\varepsilon),(11 / 68+\varepsilon, 1 / 2+$ $11 / 68+\varepsilon)[3]$,

(c) $(9 / 56+\varepsilon, 1 / 2+9 / 56+\varepsilon)[5]$,

(d) $(89 / 560+\varepsilon, 1 / 2+89 / 560+\varepsilon)[11]$,

(e) $\mathrm{H}_{05}:=(32 / 205+\varepsilon, 1 / 2+32 / 205+\varepsilon)[4]$.

2. $\mathrm{A}(\mathrm{k}, \mathrm{l}) \in \mathrm{P}$ and $\mathrm{BA}(\mathrm{k}, \mathrm{l}) \in \mathrm{P}$ for every $(\mathrm{k}, \mathrm{l}) \in \mathrm{P}$, where operators $\mathrm{A}$ and $\mathrm{B}$ are defined as follows:

$$
A(k, l)=\left(\frac{k}{2(k+1)}, \frac{k+l+1}{2(k+1)}\right), \quad B(k, l)=(l-1 / 2, k+1 / 2) .
$$

Possibly the set of the exponent pairs includes elements $(k, l) \notin$ conv $\mathrm{P}$, but at least conv $\mathrm{P}$ incorporates all currently known exponent pairs. Everywhere below writing "a set of exponent pairs" we mean $\mathrm{P}$ in fact.

Denote by $\mathrm{Pp}$ a set of exponent pairs, generated from the pair $p$ with the use of operators $A$ and BA. One can check that currently

$$
\operatorname{conv} \mathrm{P}=\operatorname{conv}\left(\mathrm{PH}_{05} \cup\{(0,1),(1 / 2,1 / 2)\}\right) .
$$

Many asymptotic questions of the number theory (especially in the area of divisor problems) come to the optimization task

$$
\inf _{(k, l) \in \operatorname{conv} P}\left\{\theta(k, l) \mid R_{i}\left(\alpha_{i} k+\beta_{i} l+\gamma_{i}\right), \quad i=1, \ldots, j\right\},
$$

where $\alpha_{i}, \beta_{i}, \gamma_{i} \in \mathbb{R}, R_{i} \in R_{>}, R_{\geq}$, predicate $R_{>}$checks whether its argument is a positive value and $R_{\geq}$checks whether its argument is non-negative, $i=$ $1, \ldots, j$.

Graham [2] gave an effective method, which in many cases is able to determine

$$
\inf _{(k, l) \in \operatorname{conv} P(0,1)} \theta(k, l)
$$


with a given precision (for certain $\theta$ - even exactly), where

$$
\theta \in \Theta:=\left\{(k, l) \mapsto \frac{a k+b l+c}{d k+e l+f} \mid \begin{array}{c}
a, b, c, d, e, f \in \mathbb{R}, \\
d k+e l+f>0 \text { for }(k, l) \in \text { conv } P
\end{array}\right\} .
$$

We shall refer to this result as to Graham algorithm. Unfortunately, for some objective functions $\theta \in \Theta$ the algorithm fails and, as Graham writes, we should "resort to manual calculations and ad hoc arguments". We discuss possible improvements in Section 4.

The primary aim of the current paper is to provide an algorithm to determine

$$
\inf _{(k, l) \in P} \theta(k, l)
$$

under a nonempty set of linear constrains (thus $j \neq 0$ ) and

$$
\theta=\max \left\{\theta_{1}(k, l), \ldots, \theta_{m}(k, l)\right\}, \quad \theta_{1}, \ldots, \theta_{m} \in \Theta .
$$

In Section 2 a useful computational concept of projective exponent pairs is explained. Section 3 is devoted to the exploration of the geometry of $\mathrm{P}$ and its results are of separate interest. In Section 4 Graham algorithm is discussed. Section 5 contains the description of the proposed algorithm to solve (2) under linear constrains and (3). In Section 6 new estimates and theoretical results on various divisor problems are given, derived from the observation of particular cases of the output of our algorithm.

\section{Projective exponent pairs}

Let us map exponent pairs into the real projective space (the concept of such mapping traces back to Graham [2]):

$$
\mu: \mathbb{R}^{2} \rightarrow \mathbb{R}^{3} /(\mathbb{R} \backslash\{0\}), \quad(k, l) \mapsto(k: l: 1) .
$$

For the set of the exponent pairs the inverse mapping

$$
\mu^{-1}:(k: l: m) \mapsto(k / m, l / m)
$$

is also well-defined.

Operators $A$ and $B A$ are mapped by $\mu$ into linear operators over projective space:

$$
\mathrm{A}(\mathrm{k}, \mathrm{l}) \mapsto \mathcal{A}(\mathrm{k}: \mathrm{l}: 1), \quad \mathcal{A}=\left(\begin{array}{lll}
1 & 0 & 0 \\
1 & 1 & 1 \\
2 & 0 & 2
\end{array}\right), \quad \mathcal{A}(\mathrm{k}: \mathrm{l}: \mathrm{m})=\left(\begin{array}{c}
\mathrm{k} \\
\mathrm{k}+\mathrm{l}+\mathrm{m} \\
2 \mathrm{k}+2 \mathrm{~m}
\end{array}\right)
$$


and

$\mathrm{BA}(\mathrm{k}, l) \mapsto \mathcal{B} \mathcal{A}(\mathrm{k}: l: 1), \quad \mathcal{B} \mathcal{A}=\left(\begin{array}{lll}0 & 1 & 0 \\ 2 & 0 & 1 \\ 2 & 0 & 2\end{array}\right), \quad \mathcal{B} \mathcal{A}(k: l: m)=\left(\begin{array}{c}l \\ 2 k+m \\ 2 k+2 m\end{array}\right)$.

Thus $A=\mu^{-1} \mathcal{A} \mu$ and $B A=\mu^{-1} \mathcal{B A} \mu$

Such projective mappings are very useful to achieve better computational performance.

Firstly, we replace fractional calculations with integer ones.

Secondly, let $M$ be a fixed composition of $A$ and $B A$. We can evaluate $M p$ for a set of points $p$ effectively: once precompute the matrix of the projective operator $\mathcal{M}$ and then just calculate $\mu^{-1} \mathcal{M} \mu$ for each point $p$.

\section{$3 \quad$ Exploring exponent pairs}

Let us split $\mathrm{Pp}$ into generations $\mathrm{P}_{\mathrm{n}} \mathrm{p}$ such that

$$
P_{0} p=\{p\}, \quad P_{n} p=A P_{n-1} p \cup B A P_{n-1} p, \quad n>0 .
$$

Let us investigate properties of $\mathrm{P}(0,1)$. As soon as

$$
\begin{aligned}
& \mathrm{A}(0,1)=(0,1), \quad \mathrm{BA}(0,1)=(1 / 2,1 / 2), \\
& \mathrm{A}(1 / 2,1 / 2)=\mathrm{BA}(1 / 2,1 / 2)=(1 / 6,2 / 3),
\end{aligned}
$$

we obtain

$$
\mathrm{P}(0,1)=\{(0,1),(1 / 2,1 / 2)\} \cup \mathrm{P}(1 / 6,2 / 3) .
$$

So it is enough to study $\mathrm{P}(1 / 6,2 / 3)$.

All initial exponent pairs satisfy inequalities

$$
k+l \leqslant 1, \quad k \leqslant 1 / 2, \quad l \geqslant 1 / 2 .
$$

One can check that if $(k, l)$ satisfies such inequalities, then $A(k, l)$ and $B A(k, l)$ also do. Thus all exponent pairs fits into the triangle

$$
\mathrm{T}:=\triangle((1 / 2,1 / 2),(0,1),(0,1 / 2)) .
$$

Lemma 3 Denote

$$
\mathrm{P}^{\prime}=(0,1 / 6) \times(2 / 3,1), \quad \mathrm{P}^{\prime \prime}=(1 / 6,1 / 2) \times(1 / 2,2 / 3) .
$$

Let $\mathrm{p}:=(\mathrm{k}, \mathrm{l})$ be the exponent pair such that $\mathrm{Ap} \in \mathrm{P}^{\prime}$. Then

$$
A P p \subset P^{\prime}, \quad B A P p \subset P^{\prime \prime}, \quad P p \subset\{p\} \cup P^{\prime} \cup P^{\prime \prime} .
$$


Proof. Suppose that (5) is true for all generations $\mathrm{P}_{\mathrm{m}}, \mathrm{m}<\mathrm{n}$. Let us prove that it is also true for generation $P_{n}$.

We have $B P^{\prime}=P^{\prime \prime}$, so it is enough to prove that $A P_{n-1} p \subset P^{\prime}$. Let $(k, l)$ be an arbitrary element of $P_{n-1} p$ and let $(\kappa, \lambda)=A(k, l)$. There are three possibilities:

1. $(k, l)=p$. Then $(\kappa, \lambda) \in \mathrm{P}^{\prime}$ by conditions of the lemma.

2. $(k, l) \in P^{\prime}$. Then

$$
\begin{gathered}
\kappa=\frac{1}{2}-\frac{1}{2(k+1)}<\frac{1}{2}-\frac{3}{7}=\frac{1}{14}, \\
\lambda=\frac{1}{2}+\frac{l}{2(k+1)}>\frac{1}{2}+\frac{2 / 3}{7 / 3}=\frac{11}{14} .
\end{gathered}
$$

3. $(k, l) \in \mathrm{P}^{\prime \prime}$. Then

$$
\begin{array}{r}
\kappa=\frac{1}{2}-\frac{1}{2(k+1)}<\frac{1}{2}-\frac{1}{3}=\frac{1}{6}, \\
\lambda=\frac{1}{2}+\frac{l}{2(k+1)}>\frac{1}{2}+\frac{1 / 2}{3}=\frac{2}{3} .
\end{array}
$$

An exponent pair $(1 / 6,2 / 3)$ satisfies conditions of Lemma 3, because

$$
A(1 / 6,2 / 3)=(1 / 14,11 / 14) .
$$

We note that the statement of Lemma 3 can be refined step-by-step, obtaining 4, 8, 16 and so on rectangles, covering Pp more and more precisely.

Remark 4 There exists another approach to cover Pp or the whole P. For a set of pairs $\left(\alpha_{i}, \beta_{i}\right)$ determine with the use of Graham algorithm

$$
\theta_{i}=\inf _{(k, l) \in \operatorname{conv} P p}\left(\alpha_{i} k+\beta_{i} l\right) .
$$

Then Pp is embedded into a polygonal area, constrained with the set of inequalities

$$
\alpha_{i} k+\beta_{i} l \geqslant \theta_{i}
$$

from the bottom and left (together they form a hyperbola-like line) and by the segment from $(0,1)$ to $(1 / 2,1 / 2)$. 
Let us introduce an order $\prec$ on $\mathrm{P}(1 / 6,2 / 3)$, defined as

$$
(k, l) \prec(\kappa, \lambda) \Longleftrightarrow k<\kappa, l>\lambda .
$$

Theorem 5 Let $\mathrm{p}$ be the exponent pair from the statement of Lemma 3. Then the order $\prec$ is a strict total order on $\mathrm{P}_{\mathrm{n}} \mathrm{p}$ and this order coincides with the order of the binary Gray codes [7, Ch. 7.2.1.1] over an alphabet $\{\mathrm{A}, \mathrm{BA}\}$.

Proof. One can directly check that operator A saves the order:

$$
\mathrm{p}_{1} \prec \mathrm{p}_{2} \Rightarrow A \mathrm{p}_{1} \prec A \mathrm{p}_{2}
$$

and operator B reverses it, so BA reverses it too:

$$
\mathrm{p}_{1} \prec \mathrm{p}_{2} \Rightarrow \mathrm{BAp}_{1} \succ \mathrm{BAp}_{2} .
$$

Lemma 3 implies that for every $p_{1}, p_{2} \in P_{n-1} p$

$$
A p_{1} \prec B A p_{2} \text {. }
$$

Combining these facts we obtain the statement of the theorem.

In the case of $\mathrm{P}(1 / 6,2 / 3)$ inequality (6) can be refined up to

$$
A p_{1} \prec(1 / 6,2 / 3) \prec B_{A p} .
$$

Thus $\prec$ is a strict total order over the whole $\mathrm{P}(1 / 6,2 / 3)$.

Fig. 1 illustrates our results. Point $(1 / 6,2 / 3)$ divides the set into rectangles $\mathrm{P}^{\prime}$ and $\mathrm{P}^{\prime \prime}$. These rectangles consists of pairs, where the last applied operator was $A$, and pairs, where the last applied operator was BA, respectively. All plotted points are total-ordered by $\prec$. Writing out points of the same generation from the left top corner to the right bottom corner we obtain a list of Gray codes. E. g., for the generation 3 we obtain a sequence of 8 codes:

A $A$ A,

A $A B A$,

ABABA,

ABA A,

BABA A,

BABABA,

BA ABA,

$B A A$ A. 


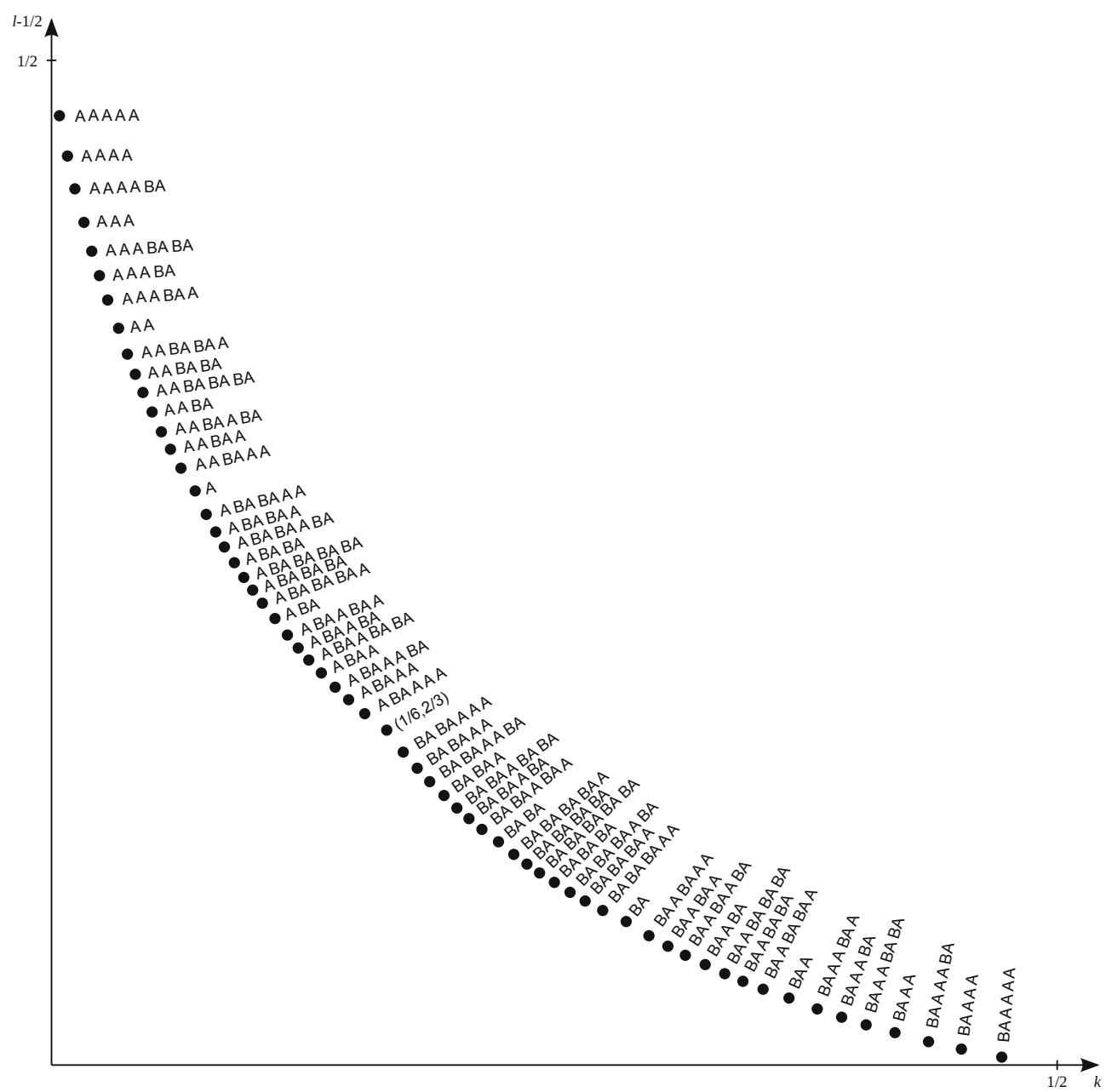

Figure 1: First six generations of $\mathrm{P}(1 / 6,2 / 3)$ plotted in shifted coordinates $(k, l-1 / 2)$. 
As soon as

$$
A^{\mathrm{n}}(1 / 6,2 / 3) \rightarrow(0+, 1-0) \quad \text { as } \mathrm{n} \rightarrow \infty
$$

we obtain

$$
\begin{aligned}
p_{n}:=A \cdot B A \cdot A^{n}(1 / 6,2 / 3) & \rightarrow(1 / 6-0,2 / 3+0), \\
B A \cdot B A \cdot A^{n}(1 / 6,2 / 3) & \rightarrow(1 / 6+0,2 / 3-0) \quad \text { as } n \rightarrow \infty .
\end{aligned}
$$

So no point from $\mathrm{P}(1 / 6,2 / 3)$ is isolated: for every $p \in \mathrm{P}(1 / 6,2 / 3)$ and every $\varepsilon>0$ there exist $p_{1}, p_{2} \in \mathrm{P}(1 / 6,2 / 3)$ such that $p_{1} \prec p \prec p_{2},\left|p-p_{1}\right|<\varepsilon$ and $\left|p-p_{2}\right|<\varepsilon$.

We are even able to compute the slopes of left-hand and right-hand "tangents" at $(1 / 6,2 / 3)$. Namely, using Section 2 and denoting $d_{n}=p_{n}-(1 / 6,2 / 3)$ we get

$$
\mathrm{d}_{\mathrm{n}} /\left|\mathrm{d}_{\mathrm{n}}\right| \rightarrow(-2 / \sqrt{5}, 1 / \sqrt{5}) \quad \text { as } \mathrm{n} \rightarrow \infty,
$$

so the left-hand "tangent" at $(1 / 6,2 / 3)$ has a slope $\arctan (-1 / 2)$. The righthand "tangent" has a slope $\arctan (-2)$.

What about sets generated from other known initial exponent pairs, listed in Proposition 2? Lemma 3 and Theorem 5 remains valid. But inequality (7) does not hold and so $\prec$ is not a strict total order. E. g., for

$$
p=A \cdot B A \cdot A^{4} H_{05}=\left(\frac{8083}{50342}, \frac{1}{2}+\frac{4304}{25171}\right)
$$

neither $\mathrm{p} \prec \mathrm{H}_{05}$, nor $\mathrm{p} \succ \mathrm{H}_{05}$.

As opposed to $P(1 / 6,2 / 3)$, each point of the set $P p, p \neq(1 / 6,2 / 3)$, is isolated, because the initial point is. But for every such $p$ each point of $P(1 / 6,2 / 3)$ has an arbitrary close to it point from Pp.

Lemma 6 Operators $\mathrm{A}$ and $\mathrm{BA}$ are contractions over the triangle $\mathrm{T}$, which was defined in (4).

Proof. It is enough to prove that $A$ is a contraction. Let us check that there exists $\alpha<1$ such that for each $p_{1}, p_{2} \in T$ we have

$$
\left|A p_{1}-A p_{2}\right| \leqslant \alpha\left|p_{1}-p_{2}\right|
$$


Let $\left(k_{1}, l_{1}\right):=p_{1}$ and $\left(k_{2}, l_{2}\right):=p_{2}$. Then

$$
\begin{aligned}
\mid A p_{1} & -\left.A p_{2}\right|^{2}=\frac{1}{4}\left(\left(\frac{1}{k_{1}+1}-\frac{1}{k_{2}+1}\right)^{2}+\left(\frac{l_{1}}{k_{1}+1}-\frac{l_{2}}{k_{2}+1}\right)^{2}\right)= \\
= & \frac{1}{4}\left(\left(\frac{k_{2}-k_{1}}{\left(k_{1}+1\right)\left(k_{2}+1\right)}\right)^{2}+\left(\frac{\left(l_{1}-l_{2}\right)\left(k_{2}+1\right)+l_{2}\left(k_{2}-k_{1}\right)}{\left(k_{1}+1\right)\left(k_{2}+1\right)}\right)^{2}\right) .
\end{aligned}
$$

But $k_{1}, k_{2} \geqslant 0$, so

$$
\left|A p_{1}-A p_{2}\right|^{2} \leqslant \frac{1}{4}\left(\left(k_{1}-k_{2}\right)^{2}+\left(\left|l_{1}-l_{2}\right|+\left|k_{1}-k_{2}\right|\right)^{2}\right) .
$$

Applying inequality $(x+y)^{2} \leqslant 2\left(x^{2}+y^{2}\right)$ we finally obtain

$$
\left|A p_{1}-A p_{2}\right|^{2} \leqslant \frac{3}{4}\left(\left(k_{1}-k_{2}\right)^{2}+\left(l_{1}-l_{2}\right)^{2}\right)=\frac{3}{4}\left|p_{1}-p_{2}\right|^{2} .
$$

\section{Notes on Graham algorithm}

Below GX means a reference to [2, Step X at p. 209].

1. Graham algorithm is designed to search $\inf _{\mathrm{p} \in \mathrm{P}(0,1)} \theta p$ and relies on the fact that

$$
\mathrm{P}(0,1)=\mathrm{AP}(0,1) \cup \operatorname{BAP}(0,1) .
$$

This kind of decomposition does not hold for the whole P. Instead we have

$$
\mathrm{P}=\mathrm{AP} \cup \mathrm{BAP} \cup\left(\mathrm{P}_{0} \backslash\{(0,1)\}\right) .
$$

Thus in order to run Graham algorithm over $\mathrm{P}$, not just over $\mathrm{P}(0,1)$, it should be changed in following way. Establish a variable $r$ to keep a current minimal value, setting it initially to $+\infty$. Add an additional step before G5: apply current $\theta$ on elements of $P_{0}$ and set $r \leftarrow \min \left(r, \min \theta P_{0}\right)$. At the end of the algorithm output $r$ instead of simply $\min \theta \mathrm{P}_{0}$.

2. Unfortunately, Graham algorithm over $P$ is infinite: no analog of halting conditions at G3 provided by [2, Th. 3] can be easily derived. So we should stop depending on whether the desired accuracy is achieved. Cf. Step 2 in the Section 5 below. 
3. Bad news: if [2, Th. 1, 2] does not specify the branch to choose at G4 then the original Graham algorithm halts. Good news: [2, Th. 2] can be generalized to cover a wider range of cases. In notations of the mentioned theorem for a given finite sequence $M \in\{A, B A\}^{n}$ if inf $\theta B A=\inf \theta B A M$ and if

$$
\min (r w+v-u, \alpha w+v-u) \geqslant 0
$$

then $\inf \theta=\inf \theta A$, where

$$
\alpha:=\max \{k+l \mid(k, l) \in \operatorname{AM}\{(0,1),(1 / 2,1 / 2),(0,1 / 2)\}\} .
$$

4. For the case of linearly constrained optimization one can build a "greedy" modification of Graham algorithm: if at G5 one of the branches is entirely out of constrains then choose another one; otherwise choose a branch in a normal way. Such algorithm executes pretty fast, but misses optimal pairs sometimes.

\section{$5 \quad$ Linear programming algorithm}

Now let us return to the optimization problem (2). We will attack it with the use of backtracking.

Operators $A$ and BA perform projective mappings of the plane $\mathbb{R}^{2}$, so both of them map straight lines into lines and polygons into polygons.

Let $\theta$ be as in (3) and a set of linear constrains LC be as in (1).

Denote

$$
\theta_{+}(\mathrm{V})=\max \left\{\sup _{\mathrm{p} \in \mathrm{V}} \theta_{i} p\right\}_{i=1}^{\mathrm{m}}, \quad \theta_{-}(\mathrm{V})=\max \left\{\inf _{\mathrm{p} \in \mathrm{V}} \theta_{i} p\right\}_{i=1}^{\mathrm{m}} .
$$

Then

$$
\theta_{-}(\mathrm{V}) \leqslant \inf _{\mathrm{p} \in \mathrm{V}} \theta p \leqslant \theta_{+}(\mathrm{V})
$$

and these bounds embrace $\inf _{p \in V} \theta p$ tighter and tighter as $\mathrm{V}$ becomes smaller. Both $\theta_{+}$and $\theta_{-}$can be computed effectively by simplex method.

Let $\mathrm{V}$ be a polygon (or a set of polygons, lines and points) such that $\mathrm{P} \subset \mathrm{V}$. See Lemma 3 and paragraphs above and below it for possible constructions of $\mathrm{V}$. For a set of linear constrains $\mathrm{LC}$ let $\mathrm{R}(\mathrm{V}, \mathrm{LC})$ be a predicate, which is true if and only if there exists a point $p \in V$, which satisfies all constrains from LC. This predicate can be computed effectively using algorithms for line segment intersections [1, p. 19-44]. 
The proposed algorithm consists of a routine $\mathrm{L}(\theta, \mathrm{LC}, \mathrm{r}, \mathcal{M})$, which calls itself recursively. Here $r$ keeps a current minimal value of $\theta(k, l)$ and $\mathcal{M}$ is a current projective transformation matrix. Initially $r \leftarrow+\infty$ and $\mathcal{M} \leftarrow\left(\begin{array}{lll}1 & 0 & 0 \\ 0 & 1 & 0 \\ 0 & 0 & 1\end{array}\right)$.

On each call routine L performs following steps:

1. Compute $t \leftarrow \min \left\{\theta \mu^{-1} \mathcal{M} \mu p\right\}$, where $p$ runs over all known initial pairs $p$. If we get $t<r$ then update current minimal value: $r \leftarrow t$.

2. Check whether the desired accuracy is achieved, comparing $r$ with the values of $\theta_{+}\left(\mu^{-1} \mathcal{M} \mu \mathrm{V}\right)$ and $\theta_{-}\left(\mu^{-1} \mathcal{M} \mu \mathrm{V}\right)$. If yes then return $r$ and abort computations.

3. Set $\mathrm{LC}^{\prime} \leftarrow \operatorname{LC} \cup\left\{\theta_{i}(k, l)<r\right\}_{i=1}^{m}$. Due to the nature of $\theta_{i} \in \Theta$ a constrain of form $\theta_{i}(k, l)<r$ is in fact a linear constrain.

4. If $\mathrm{R}\left(\mu^{-1} \mathcal{M} \mathcal{A} \mu \mathrm{V}, \mathrm{LC}^{\prime}\right)$ (that means that there is at least a chance to meet exponent pair $p \in \mu^{-1} \mathcal{M A} \mu \mathrm{V}$, which satisfies $\mathrm{LC}$ and on which objective function is less than yet achieved value) then compute $t \leftarrow$ $\mathrm{L}(\theta, \mathrm{LC}, \mathrm{r}, \mathcal{M \mathcal { A }})$. If $\mathrm{t}<\mathrm{r}$ set $\mathrm{r} \leftarrow \mathrm{t}$ and recompute $\mathrm{LC}^{\prime}$ as in Step 3 using the new value of $r$.

5. If $\mathrm{R}\left(\mu^{-1} \mathcal{M B} \mathcal{A} \mu \mathrm{V}, \mathrm{LC}^{\prime}\right)$ then compute $\mathrm{t} \leftarrow \mathrm{L}(\theta, \mathrm{LC}, \mathrm{r}, \mathcal{M B \mathcal { A }})$. If $\mathrm{t}<\mathrm{r}$ set $r \leftarrow t$.

6. Return $r$.

The algorithm executes in finite time, because due to Lemma 6 both $A$ and $B A$ are contractions and sooner or later (depending on required accuracy) recursively called routines will abort at Step 2.

Step 3 plays a crucial role in chopping off non-optimal branches of the exhaustive search and preventing exponential running time. We are not able to provide any theoretical estimates, but in all our experiments (see Section 6 below) the number of calls of $\mathrm{L}(\cdot, \cdot, \cdot, \cdot)$ behaved like a linear function of the recursion's depth.

We have implemented our algorithm as a program, written in PARI/GP [10]. It appears that it runs pretty fast, in a fraction of a second on the modern hardware.

During computations elements of $\mathcal{M}$ can grow enormously. As soon as matrix $\mathcal{M}$ is applied on projective vectors we can divide $\mathcal{M}$ on the greatest common divisor of its elements to decrease their magnitude. 
Now, under which circumstances an equality

$$
\inf _{p \in P} \theta p=\inf _{p \in \operatorname{conv} P} \theta p
$$

holds? Certainly it is true for $\theta \in \Theta$ and $j=0$, because sets $\{\theta p=$ const $\}$ are straight lines; this is the case of Graham algorithm.

Consider the case $\theta \in \Theta$ and $j \neq 0$. Constraining lines are specified by equations

$$
l_{i}=\left\{\alpha_{i} k+\beta_{i} l+\gamma_{i}=0\right\}, \quad i=1, \ldots, j .
$$

Then

$$
\inf _{p \in \operatorname{conv} P} \theta p=\min \left\{\inf _{p \in P} \theta p, \inf _{p \in l_{1} \cap \operatorname{conv} P} \theta p, \ldots, \inf _{p \in l_{j} \cap \operatorname{conv} P} \theta p\right\} .
$$

But conv $\mathrm{P}$ is approximated by a polygon as in Remark 4 , so $l_{i} \cap$ conv $\mathrm{P}$ can be approximated too and consists of a single segment. Thus $\inf _{p \in l_{i} \cap \operatorname{conv} P} \theta p$ is computable.

The case when $\theta$ is as in (3) with $m>1$ is different. Even without any constrains the value of $\inf _{p \in \operatorname{conv}} P \theta p$ may be not equal to $\inf _{p \in P} \theta p$. For example, take

$$
\theta(k, l)=\max \{11 k / 10, l-1 / 2\} \text {. }
$$

Then

$$
\inf _{p \in P} \theta p=\frac{176}{1025} \quad \text { at } p=\mathrm{H}_{05}
$$

But

$$
\inf _{p \in \operatorname{conv} P} \theta p=\frac{176}{1057} \quad \text { at } p=(160 / 1057,1409 / 2114):=q,
$$

and $\mathrm{q}$ is owned by a segment from $(0,1)$ to $\mathrm{H}_{05}$. However, in not-so-synthetic cases the proposed algorithm produces results, which are closer to optimal.

\section{Applications}

One can run algorithm from the previous section to obtain numerical results in partial cases for different objective functions and constrains. It gives us a way to catch site of some patterns and to suppose general statements on them. Nevertheless these patterns should be proved, not only observed. This is the main theme of the current section. 
Consider the asymmetrical divisor problem. Denote

$$
\tau\left(a_{1}, \ldots, a_{k} ; n\right)=\sum_{d_{1}^{a_{1} \ldots d_{k}^{a_{k}}=n}} 1,
$$

which is called an asymmetrical divisor function. Let $\Delta\left(a_{1}, \ldots, a_{m} ; x\right)$ be an error term in the asymptotic estimate of the sum $\sum_{n \leqslant x} \tau\left(a_{1}, \ldots, a_{m} ; n\right)$. (See [8] for the form of the main term.) What upper estimates of $\Delta$ can be given? The following result is one of the possible answers.

Theorem $7([8$, Th. 5.11] $)$ Let $\mathrm{a}<\mathrm{b}$ and let $(\mathrm{k}, \mathrm{l})=\mathrm{A}(\mathrm{\kappa}, \lambda)$ be an exponent pair. Then the estimate

$$
\Delta(\mathrm{a}, \mathrm{b} ; \mathrm{x}) \ll x^{\alpha} \log x, \quad \alpha=\frac{2(k+l-1 / 2)}{(\mathrm{a}+\mathrm{b})}
$$

holds under the condition $(2 \mathrm{l}-1) \mathrm{a} \geqslant 2 \mathrm{~kb}$. Here $\mathrm{f}(\mathrm{x}) \ll \mathrm{g}(\mathrm{x})$ denotes $\mathrm{f}(\mathrm{x})=$ $\mathrm{O}(\mathrm{g}(\mathrm{x}))$. If otherwise $(2 \mathrm{l}-1) \mathrm{a}<2 \mathrm{~kb}$, then

$$
\Delta(\mathrm{a}, \mathrm{b} ; \mathrm{x}) \ll x^{\alpha} \log x, \quad \alpha=\frac{k}{(1-l) a+k b} .
$$

Taking into account Lemma 3 the condition $(k, l)=A(\kappa, \lambda)$ can be rewritten as $k<1 / 6$ and $l>2 / 3$. Thus

$$
\begin{array}{cl}
\theta_{1}=\frac{2(k+l-1 / 2)}{a+b}, & \operatorname{LC}_{1}=\{(2 l-1) a \geqslant 2 k b, k<1 / 6, l>2 / 3\}, \\
\theta_{2}=\frac{k}{(1-l) a+k b}, & \operatorname{LC}_{2}=\{(2 l-1) a<2 k b, k<1 / 6, l>2 / 3\} .
\end{array}
$$

Using proposed algorithm we can compute inf $\theta_{1}$ under constrains LC $_{1}$ (which refers to the first case of Theorem 7), compute inf $\theta_{2}$ under constrains $L_{2}$ (which refers to the second case) and take lesser of the obtained values. Observed results shows that for $a=1, b=2^{r}, r \geqslant 10$, the second case provides better results and exponent pair has form

$$
A^{r-1} B^{-1} A^{r-4} B A B A \ldots
$$

This leads us to the following statement.

Theorem 8 For a fixed integer $r \geqslant 5$ we have $\Delta\left(1,2^{r} ; x\right) \ll \chi^{\alpha} \log x$, where

$$
\alpha=\frac{2^{r}-2 r}{2^{2 r}-r \cdot 2^{r}-2 r^{2}+2 r-4}<\frac{1}{2^{r}+r} .
$$


Proof. Consider an exponent pair

$$
\left(k_{r}, l_{r}\right):=A^{r-1} \operatorname{BAA}^{r-4}(1 / 6,2 / 3) .
$$

We have

$$
\mathcal{A}=\mathcal{S}\left(\begin{array}{lll}
1 & 1 & 0 \\
0 & 1 & 0 \\
0 & 0 & 2
\end{array}\right) \mathcal{S}^{-1}, \quad \mathcal{S}=\left(\begin{array}{ccc}
0 & -1 & 0 \\
1 & 0 & 1 \\
0 & 2 & 1
\end{array}\right)
$$

Thus $\mathcal{A}^{\mathfrak{n}}=\mathcal{S}\left(\begin{array}{ccc}1 & n & 0 \\ 0 & 1 & 0 \\ 0 & 0 & 2^{\mathrm{n}}\end{array}\right) \mathcal{S}^{-1}$. Note that $\mu(1 / 6,2 / 3)=(1: 4: 6)$ and

$$
\mathcal{A}^{\mathrm{r}-1} \mathcal{B} \mathcal{A} \mathcal{A}^{\mathrm{r}-4}(1: 4: 6)=\left(\begin{array}{c}
2^{\mathrm{r}}-2 \mathrm{r} \\
2^{2 \mathrm{r}+1}-(3 \mathrm{r}+4) \cdot 2^{\mathrm{r}}+2 \mathrm{r}^{2}+2 \mathrm{r}+4 \\
2^{2 \mathrm{r}+1}-(2 \mathrm{r}+4) \cdot 2^{\mathrm{r}}+4 \mathrm{r}
\end{array}\right) .
$$

Applying $\mu^{-1}$ we get

$$
k_{r}=\frac{2^{r}-2 r}{2^{2 r+1}-(2 r+4) \cdot 2^{r}+4 r}, \quad l_{r}=1-\frac{r \cdot 2^{r}-2 r^{2}+2 r-4}{2^{2 r+1}-(2 r+4) \cdot 2^{r}+4 r} .
$$

Now for $r \geqslant 5$

$$
2 l_{r}-2 \cdot 2^{r} k_{r}-1=\frac{2 r^{2}+4-2^{r+1}}{2^{2 r}-(r+2) \cdot 2^{r}+2 r}<0 .
$$

This proves that $\left(k_{r}, l_{r}\right)$ satisfies the second case of Theorem 7 and finally

$$
\alpha=\frac{k_{r}}{2^{r} k_{r}-l_{r}+1} .
$$

In the same manner one can estimate $\Delta\left(a, 2^{r} ; x\right)$ for odd $a$. Here is one more example.

Theorem 9 For a fixed integer $r \geqslant 1$ we have $\Delta\left(3,2^{r} ; x\right) \ll x^{\alpha+\varepsilon}$, where

$$
\alpha=\frac{1}{2^{r}+3 r-88 / 17}
$$

Proof. Consider an exponent pair $(k, l):=A^{r-3} B A A(9 / 56+\varepsilon, 37 / 56+\varepsilon)$.

In the case of $\Delta(a, b, c)$ one can derive objective function and constrains from $[8$, Th. 6.2, 6.3] and observe the output of the algorithm. 


$\begin{array}{cccc}a & b & (k, l) & \Xi(a, b) \\ 1 & 2 & \mathrm{BAH}_{05} & 269 / 1217 \\ 1 & 3 & (\mathrm{BA})^{2} \mathrm{ABAH} \mathrm{H}_{05} & 1486 / 8647 \\ 1 & 4 & \mathrm{H}_{05} & 111 / 790 \\ 1 & 5 & \mathrm{ABA} \mathrm{A}^{2} \mathrm{BAA}(\mathrm{BA})^{2} \mathrm{~A}^{2} \mathrm{M}^{\infty}(0,1) & (15921-2 \mathrm{c}) / 30437 \\ 1 & 6 & (\mathrm{ABA})^{3}(\mathrm{BA})^{3} \mathrm{~A}^{3} \mathrm{BA}(0,1) & 669 / 6305 \\ 1 & 7 & \mathrm{~A}(\mathrm{BA})^{2} \mathrm{BAA}(\mathrm{BA})^{2} \mathrm{~A}^{2} \mathrm{M}^{\infty}(0,1) & (9370-\mathrm{c}) / 34469 \\ 1 & 8 & \mathrm{~A}(\mathrm{BA})^{4}\left(\mathrm{~A}^{2} \mathrm{BAA}\right)^{\infty}(0,1) & (5+\sqrt{809}) / 392 \\ 1 & 9 & \mathrm{~A}(\mathrm{BA})^{2} \mathrm{AM} \mathrm{M}^{\infty}(0,1) & (10551-\mathrm{c}) / 56976 \\ 1 & 10 & \mathrm{~A}(\mathrm{BA})^{2}\left(\mathrm{~A}^{2}(\mathrm{BA})^{2}\right)^{2} \mathrm{ABAH} \mathrm{H}_{05} & 150509 / 2096993 \\ 2 & 3 & \mathrm{BAA}(\mathrm{BA})^{2} \mathrm{~A}^{2} \mathrm{M}^{\infty}(0,1) & (\mathrm{c}-4047) / 15688 \\ 2 & 4 & \mathrm{BAH} \mathrm{H}_{05} & 269 / 2434 \\ 2 & 5 & \mathrm{M}{ }^{\infty}(0,1) & (\mathrm{c}-4311) / 18672 \\ 3 & 4 & \mathrm{BAAH} \mathrm{H}_{05} & 1819 / 19369 \\ 3 & 5 & \mathrm{BAA}(\mathrm{BA})^{3} \mathrm{~A}^{2}(\mathrm{BA})^{3} \mathrm{~A}(\mathrm{BA})^{5} \mathrm{~A}^{2} \mathrm{BA}(0,1) & 63916 / 774807 \\ 4 & 5 & \mathrm{BAAH} \mathrm{H}_{05} & 1819 / 24903\end{array}$

Table 1: Estimates of $\Xi$. Here $M=(B A)^{6}(A B A)^{2} B A A^{2}$ and $c=\sqrt{37368753}$.

Theorem 10 For a fixed integer $r \geqslant 10$ we have

$$
\theta\left(1,2^{r}, 2^{r}\right)=\frac{26 \cdot 2^{2 r}-(29 r+41) 2^{r}+16 r^{2}+12 r+32}{26 \cdot 2^{3 r}-(16 r+41) 2^{2 r}+(24 r-3) 2^{r}+16 r+12}<\frac{1}{2^{r}+1} .
$$

Proof. Follows from $\left[8\right.$, Th. 6.2] with $(k, l)=A^{r-1} B^{r-2} B A B A^{2} \cdot B(0,1)$.

Finally, consider the asymmetric divisor problem with congruence conditions on divisors. Namely, let $\tau\left(a, m_{a}, r_{a} ; b, m_{b}, r_{b} ; n\right)$ be the number of $\left(d_{a}, d_{b}\right)$ such that

$$
\mathrm{d}_{\mathrm{a}}^{\mathrm{a}} \mathrm{d}_{\mathrm{b}}^{\mathrm{b}}=\mathrm{n}, \quad \mathrm{d}_{\mathrm{a}} \equiv \mathrm{r}_{\mathrm{a}} \quad\left(\bmod \mathrm{m}_{\mathrm{a}}\right), \quad \mathrm{d}_{\mathrm{b}} \equiv \mathrm{r}_{\mathrm{b}} \quad\left(\bmod \mathrm{m}_{\mathrm{b}}\right) .
$$

Menzer and Nowak showed in [9] that if $\mathrm{a}<\mathrm{b}$ then the error term in the asymptotic estimate of

$$
\sum_{n \leqslant x} \tau\left(a, m_{a}, r_{a} ; b, m_{b}, r_{b} ; n\right)
$$

has form $\left(x / m_{a}^{a} m_{b}^{b}\right)^{\Xi(a, b)+\varepsilon}$, where

$$
\Xi(a, b):=\inf _{(k, l) \in \operatorname{conv} P} \max \left\{\frac{k+l}{(k+1)(a+b)}, \frac{k}{k b+a(1+k-l)}\right\},
$$




$\begin{array}{cccc}\sigma & \mu(\sigma) & \text { Depth 100 } & \text { Depth } 1000 \\ 3 / 5 & 1409 / 12170 & 10 & 10 \\ 2 / 3 & 0.0879154 & 154 & 1609 \\ 3 / 4 & 0.0581840 & 154 & 1610 \\ 4 / 5 & 0.0422535 & 103 & 1003\end{array}$

Table 2: Estimates for $\mu(\sigma)$ and the number of calls to L.

where $\varepsilon>0$ is arbitrary small. They also listed estimates of $\Xi(a, b)$ for $1 \leqslant$ $a<b \leqslant 5$. As soon as $\Xi(a, b)$ is of form (3) we can refine all their results. See Table 1 .

Various estimates of the Riemann zeta function depends on optimization tasks (1). The following theorem seems to be the simplest example.

Theorem $11([6,(7.57)])$ Let $\zeta$ denote the Riemann zeta function and $\sigma \geqslant$ $1 / 2$. Further, let $\mu(\sigma)$ be an infimum of all $x$ such that $\zeta(\sigma+i t) \ll t^{x}$. Then

$$
\mu(\sigma) \leqslant \frac{k+l-\sigma}{2} .
$$

for every exponent pair $(k, l)$ such that $l-k \geqslant \sigma$.

Better results on $\mu$ leads to better estimates for power moments of $\zeta$, and the last are helpful to improve estimates in multidimensional divisor problem. See $[6$, Th. 8.4, 13.2, 13.4].

Table 2 contains several results on $\mu(\sigma)$ obtained with the use of the proposed algorithm. Results are accompanied with the number of calls to $\mathrm{L}(\cdot, \cdot, \cdot, \cdot \cdot)$ up to the given depth of search.

\section{References}

[1] M. de Berg, O. Cheong, M. van Kreveld, M. Overmars, Computational Geometry: Algorithms and Applications (3rd edition), Springer, Berlin, Heidelberg, 2008. $\Rightarrow 280$

[2] S. W. Graham, An algorithm for computing optimal exponent pair, J. Lond. Math. Soc. 33, $2(1986)$ 203-218. $\Rightarrow 272,273,279,280$

[3] M. N. Huxley, Exponential sums and the Riemann zeta function, Proc. International Number Theory Conference, Laval, Canada, 1987, pp. 417-423. $\Rightarrow 272$

[4] M. N. Huxley, Exponential sums and the Riemann zeta function V, Proc. Lond. Math. Soc. 90, 1 (2005) 1-41. $\Rightarrow 272$ 
[5] M. N. Huxley, N. Watt, Exponential sums and the Riemann zeta function, Proc. Lond. Math. Soc. 57, 1 (1988) 1-24. $\Rightarrow 272$

[6] A. Ivić The Riemann Zeta-function: Theory and Applications, Dover Publications, Mineola, NY, 2003. $\Rightarrow 286$

[7] D. E. Knuth, The Art of Computer Programming. Vol. 4A. Combinatorial Algorithms, part 1, Addison-Wesley, Upper Saddle River, NJ, 2011. $\Rightarrow 276$

[8] E. Krätzel. Lattice Points, Kluwer, Dordrecht, Boston, London, 1988. $\Rightarrow 271$, 272, 283, 284, 285

[9] H. Menzer, W. G. Nowak, On an asymmetric divisor problem with congruence conditions, Manuscr. Math. 64, 1 (1989) 107-119. $\Rightarrow 285$

[10] The PARI Group, Bordeaux, PARI/GP, Version 2.6.1, 2013. $\Rightarrow 281$

[11] N. Watt, Exponential sums and the Riemann zeta function II, J. Lond. Math. Soc. 39, 3 (1989) 385-404. $\Rightarrow 272$

Received: August 28, 2013 • Revised: December 30, 2013 